
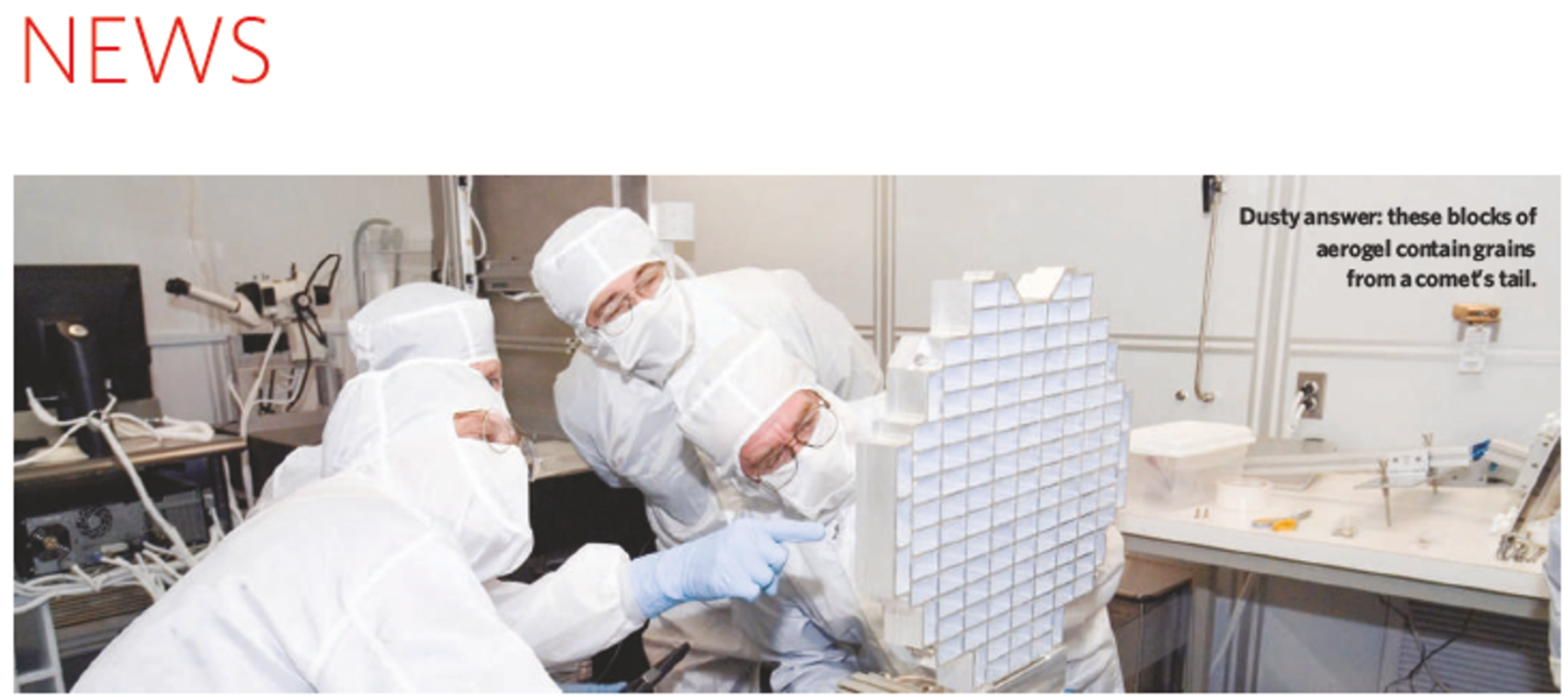

\title{
Comet chasers get mineral shock
}

\section{LEAGUE CITY, TEXAS}

The first results from a mission to catch dust from a comet's tail have revealed a surprise: these balls of dirty snow are born of fire as well as ice. Scientists were stunned to find a huge range of minerals in the particles captured by NASA's Stardust probe as it swooped past the comet Wild 2 on 2 January 2004. Many of the compounds could only have formed close to a star - far from the chilly outskirts of the Solar System where the comet first coalesced.

The Stardust team presented its preliminary results to a packed room of more than $600 \mathrm{sci}$ entists at the Lunar and Planetary Science Conference in League City, Texas, on 13 March. The grains are the first material ever brought back from a comet (see 'Caught in the wild'). Indeed, they are the first geological samples collected from space since the Soviet Luna 24 mission brought back Moon rocks two decades ago.

Of the $\mathbf{3 0}$ or so microscopic grains studied so far, almost a quarter are of a type known as calcium-aluminium inclusions (CAIs). These solidify at several thousand degrees and have never been detected in remote observations of comets. ${ }^{\alpha}$ When these grains first formed they were white hot," says Don Brownlee of the University of Washington, Seattle, who is the mission's chief scientist.

One explanation for the grains' presence is that they were created very close to the young Sun, and then flung to the outer reaches of the dusty cloud that spawned the planets. If that is the case, planetary scientists have a lot of rethinking to do - it would mean that the primitive Solar System saw a lot more mixing than previously believed.

The presence of CAIs was predicted by one controversial theory, however. The ' $\mathrm{X}$-wind' model sees strong magnetic fields around the young Sun channelling heated material to the far reaches of the protoplanetary cloud. The Stardust results do not prove anything, but they certainly fit with the idea, says Mike Zolensky of NASA's Johnson Space Center in Houston, Texas, who is leading the mineral analyses.

The other possible source of the minerals is another star completely. Once formed, the grains may have drifted through interstellar space for eons, before reaching our own Solar System and being incorporated into Wild 2 . Zolensky says it is too early to know which theory is right. But Brownlee adds that measurements of isotopes in the grains could settle the question within months - such materials often bear a distinctive isotopic signature determined by their star of origin.

Scientists involved in the work are jubilant about the progress so far. Last month, Nature spent a day with Phil Bland, a planetary scientist at Imperial College London, as he and his team analysed part of a grain (www.nature.com/ news/2006/060213/full/060213-2.html).

\section{Caught in the wild}

The 5-kilometre-wide Wild 2 was first seen in 1978 by Swiss astronomer Paul Wild. It was chosen for the Stardust mission because it spent billions of years orbiting the Sun at around the same distance as Uranus. At this distance it would have been relatively unaffected by the Sun's heat, sothere is a chance that its chemical make-up has been virtually unchanged since it was born. In 1974, a close encounter with Jupiter spun the comet towards the Sun; it now spends some of its orbit even closer to the Sun than Mars, making it relatively accessible to a visiting spacecraft.
Nature was asked by NASA not to report the results at the time, but Bland was excited to find large amounts of calcium. He speculated that it might be present in the form of calcium carbonate - even making a bet with his colleague Matt Genge. This mineral almost always forms in water, and would imply that the comet had been heated enough for its ice to melt, perhaps by collisions with meteorites.

Bland's sample has now been shown to be a CAI, and NASA scientists have not yet found any carbonates in their grains. So Bland owes Genge dinner, but he is still overjoyed to have found hints of the comet's fiery past.

And he is not the only one. "Stardust has exceeded all my expectations", says Peter Tsou of NASA's Jet Propulsion Laboratory in Pasadena, California. Tsou developed the technique of catching comet grains in an aerogel, an extremely lightweight, glass-like substance, and he has been overseeing efforts to extract dust from the carrot-shaped impact tracks. ${ }^{\alpha}$ This has been my dream for 25 years, ${ }^{n}$ he says.

Tsou adds that the success of Stardust vindicates the sometimes maligned 'faster, better, cheaper' approach to NASA missions in the 90 s, which was designed to focus on low-budget missions over short timescales. Some scientists fear that such missions are now in danger of being overlooked in favour of giant projects such as the James Webb Space Telescope (see Nature 440,140-143; 2006), especially in a NASA budget that is feeling the strain of the shuttle and International Space Station programmes.

In the meantime, work on the grains continues. Thousands of particles have yet to be analysed, says Brownlee, and "we could easily work for a year on a single particle.

MarkPeplow 